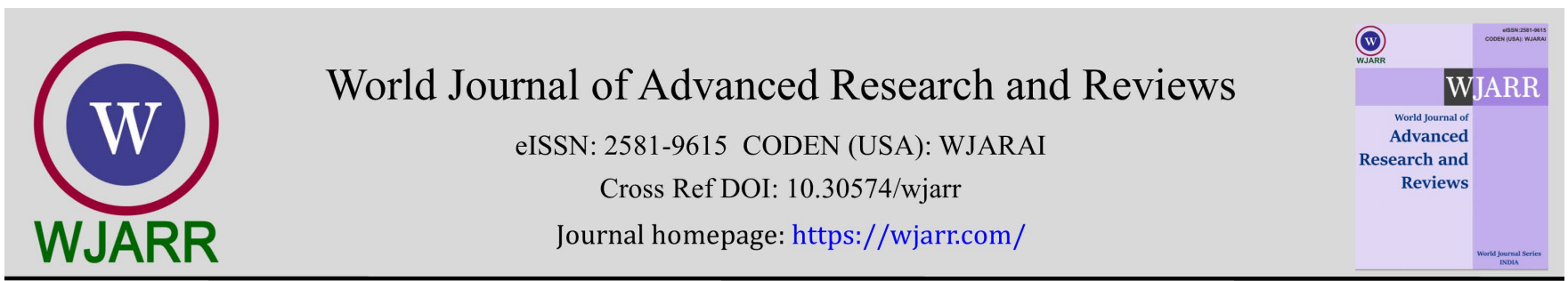

(RESEARCH ARTICLE)

\title{
Audit on induction time delays in operating room at Kibogora District Hospital: A quality improvement project
}

\author{
Jean Claude Uwimana * \\ Anesthesiologist Kibogora District hospital, Rwanda.
}

World Journal of Advanced Research and Reviews, 2021, 09(02), 163-168

Publication history: Received on 09 January 2021; revised on 12 February 2021; accepted on 14 February 2021

Article DOI: https://doi.org/10.30574/wjarr.2021.9.2.0049

\begin{abstract}
Background: Induction time delays in Operating room (OR) is an issue that affects the productivity of an operating unit especially in a setting with limited resources. It can also results in providing inappropriate services to the patients and their families. The aim of this study was to determine the causes of induction time delays and to propose solutions on how to avoid the reasons of delays.

Methods: A prospective observational study was conducted. It focused on elective general surgeries and orthopedic surgeries as they were mainly being performed during the study period. The data on the type of operation, the type of anesthesia, delay or no delay of induction (DOI) of anesthesia, causes of DOI were collected. DOI was considered as the time between the previous patient out of the OR and the next one in of more than 30 minutes. Emergency surgeries and elective obstetric surgeries were excluded from the study.

Results: $24.8 \%$ of surgeries were done after delays of induction of anesthesia as opposed to $75.2 \%$ surgeries for which anesthesia was induced without delay. $48.6 \%$ of delays of induction to anesthesia were due to the hospital issues followed by anesthesia provision related issues. (40.0\%). The surgery related and patient related issues accounted each one $5.7 \%$
\end{abstract}

Conclusions: There was a high rate of surgeries that had delays in induction times. The OR managers need to work more with the hospital administration and the OR team to correct causes of delays.

Keywords: Induction time; Delays; Causes; Operating room

\section{Introduction}

Perioperative managers and directors are under pressure to make the most of their department's resources [1]. There is an increasing interest in providing an "efficient" anaesthetic and surgical service [2,3] to make operations the largest potential source of income [2]. To use hospital resources most efficiently, reorganization of all economically relevant key processes is necessary. Because of its high cost intensity, the productivity of an operating unit of a hospital is an important [4].

Recent studies indicate that OR performance measures, such as utilization, overtime, and on-time start performance are well below achievable targets at most hospitals. Therefore they offer the potential for significant improvements [5]. Accurate scheduling of operations, matching needs with availability of room and staff, and efficient utilization of the surgical unit can provide appropriate and accessible service to patients even with limited resources [6]. Delays and consequent cancellations of surgical procedures are arguably an issue of health care quality [2].They prolong the

\footnotetext{
${ }^{*}$ Corresponding author: Jean Claude Uwimana

M.D, anesthesiologist, KIBOGORA district hospital.
}

Copyright (C) 2021 Author(s) retain the copyright of this article. This article is published under the terms of the Creative Commons Attribution Liscense 4.0. 
duration of hospitalization causing anxiety, frustration, anger, emotional involvement and inconvenience to patients and their families $[2,7,15]$. Cancellation of surgery leads to waste of time for the surgeon and other support staff as well as underutilization of operation theatre $[8,12]$.

The purpose of this study was to determine the causes of induction time delays and to propose solutions on how to avoid the reasons of delays.

\section{Material and methods}

This was an observational study performed prospectively at KIBOGORA district hospital from February 2020 to June 2020. The hospital has 3 ORs: One OR is for orthopedic surgery, the other room is for general surgery and the last one is for obstetric surgeries. The study focused on elective general surgeries and orthopedic surgeries as general surgeries and orthopedic surgeries were mainly being performed at KIBOGORA district hospital during the study period. The scheduled surgeries were performed from Monday to Friday except public holidays. The data on the date of operation, the type of operation, the type of anesthesia, delay or no DOI of anesthesia, causes of DOI whether related to the anesthesia, related to the patient or related to the surgery if DOI of anesthesia occurred. And details on the reason of delays were collected. In this study, DOI was considered as the time between the previous patient out of the OR and the next one in of more than 30 minutes. Emergency surgeries and elective obstetric surgeries were excluded from the study.

\subsection{Ethics}

The study was considered departmental audit under quality improvement project and thus it did not require ethical approval.

\subsection{Statistics}

The data collected were entered and analyzed in excel sheet. Types of anesthesia, delay versus no DOI of anesthesia distribution, causes of DOI of anesthesia and detailed reasons of delays were determined.

\section{Results}

There were 150 patients operated during the study period and 9 of them had incomplete data and were thus excluded from the data analysis. General surgeries (56.0\%) were the most surgeries done and $44.0 \%$ of the remaining ones were orthopedic surgeries as shown in the figure below. (Figure 1).

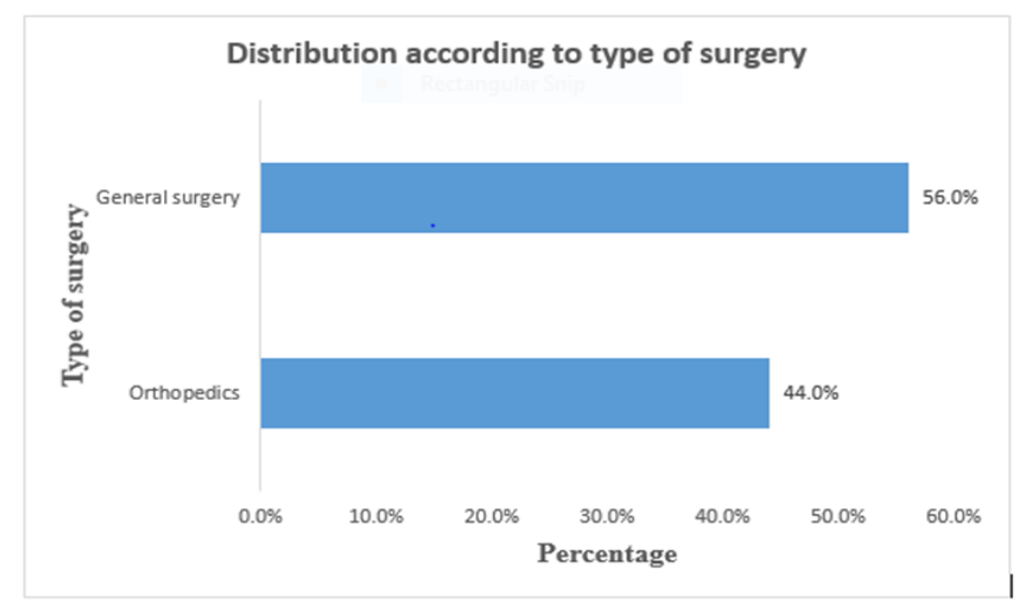

Figure 1 Distribution according to the type of surgery

In our study, 53(37.6\%), 79(56.0\%) and 9(6.4\%) patients were operated respectively under general anesthesia, spinal anesthesia and sedation as shown in figure below. (Figure 2). 


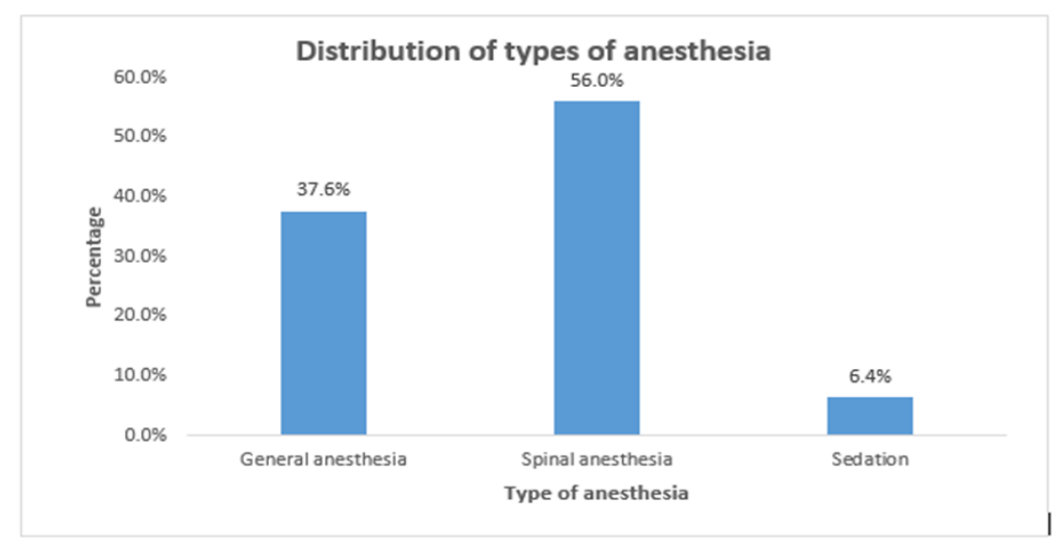

Figure 2 Distribution of type of anesthesia

During the study period, 35(24.8\%) of surgeries were done after delays of induction of anesthesia as opposed to 106 $(75.2 \%)$ surgeries for which anesthesia was induced without delay. The timing of induction (delay versus no delay) is presented in the figure below. (Figure 3).

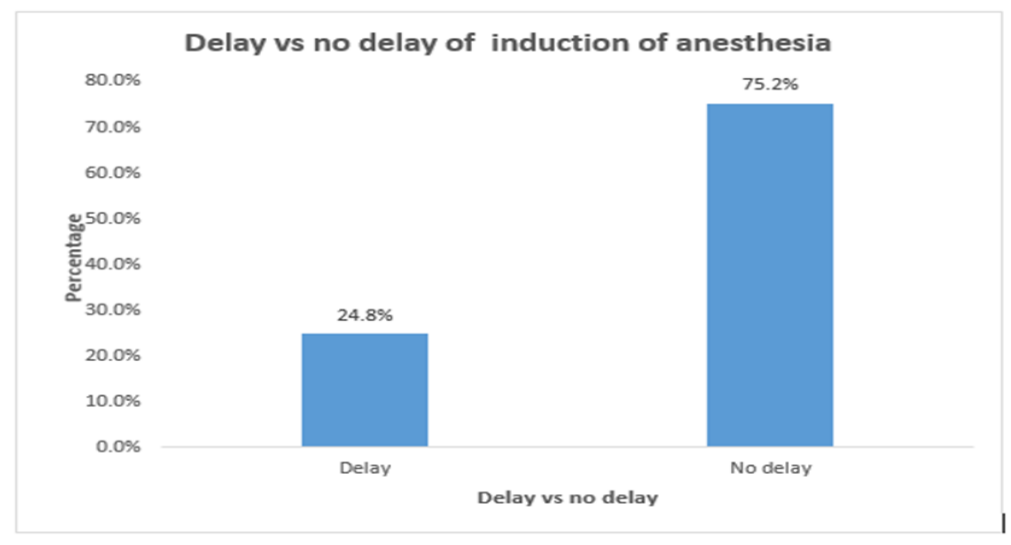

Figure 3 Distribution of timing of anesthesia

As presented in figure below, (figure 4) most causes of delays of induction to anesthesia were due to the hospital issues $(48.6 \%)$ followed by anesthesia provision related issues. $(40.0 \%)$. The surgery related and patient related issues accounted each one $5.7 \%$.

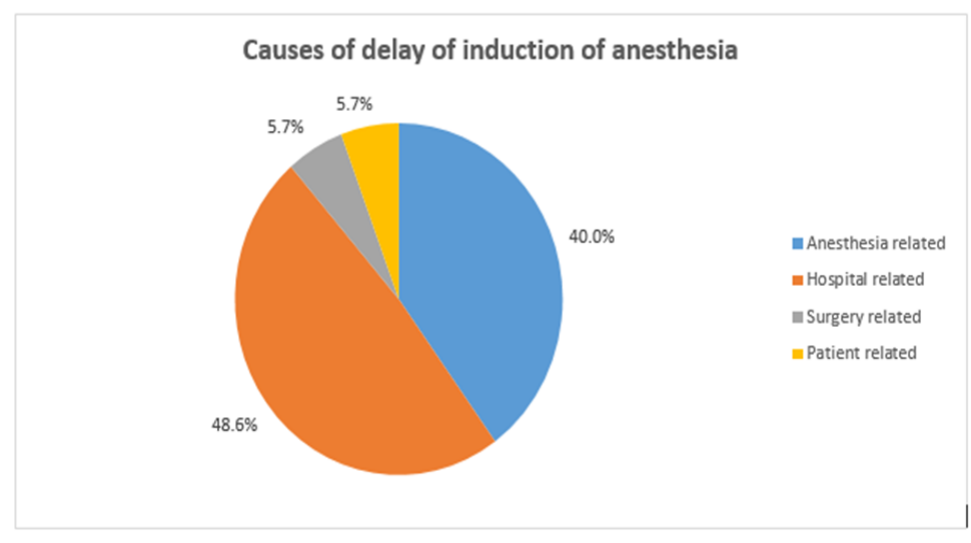

Figure 4 Causes of DOI of anesthesia

A great number $(57.1 \%)$ of reasons of delays of induction of anesthesia were avoidable whereas $42.9 \%$ were non avoidable reasons of delays of induction of anesthesia as shown in the figure below. (Figure 5) 


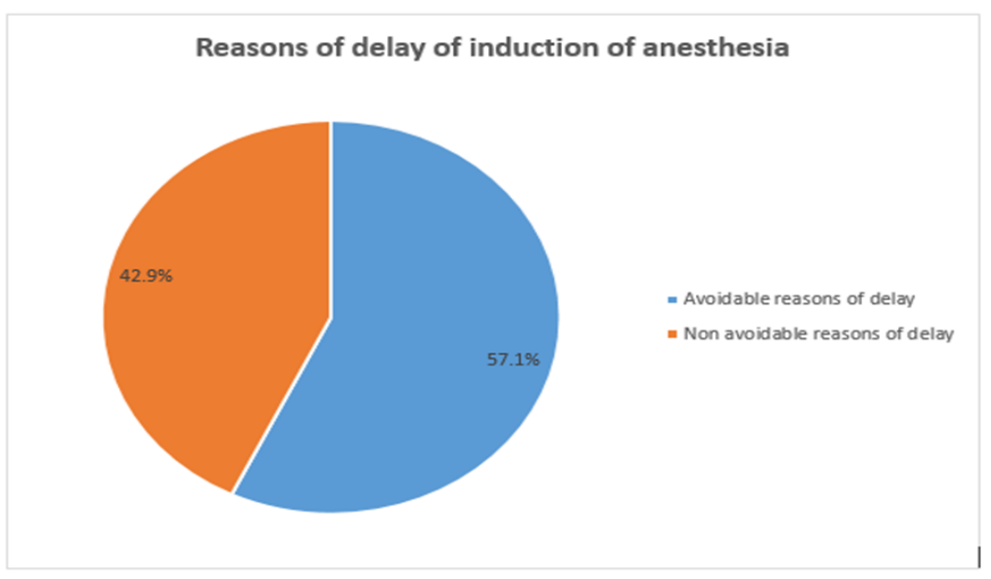

Figure 5 Reasons of DOI of anesthesia

The table below (table 1) shows details of avoidable and non-avoidable reasons of delays of induction of anesthesia.

Table 1 Avoidable and non-avoidable reasons of delays of induction of anesthesia

\begin{tabular}{|l|l|}
\hline Avoidable reasons of delays & Non avoidable reasons of delays \\
\hline Unavailability of consent forms & Medical student performing a procedure( spinal anesthesia) \\
\hline Lengthy hospital meeting & Patient was absent \\
\hline Insufficient staff & Difficult spinal \\
\hline Unavailability of equipment(stretcher) & Difficult Iv line \\
\hline Axillary block not adequate & Failed spinal anesthesia then reinjection \\
\hline Surgeon unavailability & Difficult intubation \\
\hline Infrastructure problem & Desired spinal block level achieved late \\
\hline Unavailability of essential supply & \\
\hline
\end{tabular}

\section{Discussion}

In a setting with limited resources like KIBOGORA district hospital, effective use of resources including ORs resources is of vital importance. Delays in induction times not only can lead to patient dissatisfaction but also can lead to financial constraints to the hospital. ORs are expensive and capacity limiting facilities in hospitals. Their optimal utilization is key to efficient hospital management [9].

The frequent changes in resources, objectives, and methods of health provision in contemporary medical systems necessitate repeated assessment of all its aspects [6].And the efficient and smooth running of the ORs helps control total cost of the procedure and standardization of the OR booking time [10].

We conducted this audit to analyze the reasons of delays of induction of anesthesia and whether they can be corrected. Avoiding delays in induction time will help to facilitate smooth running of the ORs, completion of the cases within allotted time and efficient time management of OR staff including surgeons, anesthetist and technicians [10].

In our study, we found a high rate of surgeries for which there were delays in induction times. $24.8 \%$ of surgeries were done after delays in induction of anesthesia as opposed to $75.2 \%$ of surgeries for which anesthesia was induced without delay. The aim of every hospital administration is to maximize utilization of OR and quick turnovers are among one of the ways to improve the use of ORs [11], the plan is to decrease the rate of induction time's delays up to less than $10 \%$ and avoid inefficient use of OR resources.

In this present study, $48.6 \%$ of the reasons for delays of induction time were related to the hospital issues followed by anesthesia provision related issues at $40.0 \%$. Surgery and patient related reasons of induction time' delays accounted $5 \%$ for each ones. Some of our findings were similar to the other ones from a study done by Kumar and Malhotra in Bangladesh where $50.5 \%$ reasons of delays in turnaround times were hospital related factors [11].The findings from 
our study show that working closely with the hospital administration to correct the reasons of delays in induction time related to the hospital issues is paramount. In this study, among the reasons of delays in induction time, 57.1\% were avoidable and $42.9 \%$ were non-avoidable. Some of the avoidable reasons of delays were lengthy hospital meetings, unavailability of equipments and supplies and insufficient hospital staff. In their study done on reasons for delay in turnover time, Kumar and Malhotra found that equipment failure, infrastructure problems and unavailability of supporting staff were among the reasons of delays [11] which was similar to our findings. Equipment failure was also one of the causes of delays that has been found in a study on delays in the OR done in Canada by Janice Wong et al. The same problem on equipments in addition to limited availability of trained supporting support were among the reasons of delays that Babita Gupta et al. found in the study they conducted on study time delays in OR [13] which is similar to what we have found in our study.

Although there were non-avoidable reasons of delays of induction time of anesthesia like when a medical student is performing a procedure, anticipation could have helped to decrease delays of induction time. Every minute that is wasted correlates to financial loss and longer waiting time for patients and physicians [16].

There are some limitations to our study. The study didn't include gynecological or obstetric patients. The present study didn't include emergencies despite the fact a great number of emergencies are being carried at KIBOGORA district hospital.

\section{Conclusion}

In this present study, a high rate $(24.8 \%)$ of surgeries had delays in induction times. There is a need to correct avoidable causes of delays. The ORs managers need to work with the hospital administration to rectify hospital related causes of delays in induction times. Anesthetists need to keep in mind that induction has to be done within the benchmarking limits. They have to avoid unnecessary delays and allocate more time ahead of the expected induction time especially when teaching students on how to perform a procedure.

\section{List of abbreviations}

OR: Operating room

DOI: Delay of induction

\section{Compliance with ethical standards}

\section{Acknowledgments}

Ndayegamiye Gilbert, Anesthetist, Kibogora district hospital for his encouragements.

\section{Disclosure of conflict of interest}

The author declare no competing interests.

\section{Statement of informed consent}

Informed consent was obtained from all individual participants included in the study."

\section{References}

[1] Foster T. Data for benchmarking your OR's performance. OR Manager. 2012; 28:13-16.

[2] Stavrou, George \& Panidis, Stavros \& Tsouskas, John Tsaousi, Georgia \& Kotzampassi, Katerina. An Audit of OR Time Utilization in a Teaching Hospital: Is There a Place for Improvement?ISRN surgery. 2014.

[3] JJ Pandit et al. Is 'starting on time'useful (or useless) as a surrogate measure for 'surgical theatre efficiency'? Anaesthesia. 2012; 67: 823-832.

[4] Hanss R, Buttgereit B, Tonner P, Bein B, Schleppers A. Steinfath Overlapping induction of anesthesia. An Analysis of Benefits and Costs. Anesthesiology. 2005; 103: 391-400. 
[5] Denton b, viapiano $j$, vogl A. Optimization of surgery sequencing and scheduling decisions under uncertainty. Health care manage sci. 2007; 10: 13-24.

[6] Avi A, Weinbroum, Ekstein P, Tiberiu E. Efficiency of the operating room suite. American journal of surgery. 2003; 185: 244-250.

[7] Ilango Ganesan, Anuradha R, Ravindrakumar P. "Audit of Cancellation of Elective Surgeries in a Teaching Hospital in South India". J of Evolution of Med and Dent. 2015; 4, 5322-5328.

[8] Bathla S, Mohta A, Gupta A, Kamal G. Cancellation of elective cases in pediatric surgery: An audit. J Indian Assoc Pediatr Surg. 2010; 15: 90-92.

[9] Does R, Vermaat T, Verver J, Bisgaard S, Heuvel J. Reducing Start Time Delays in Operating Rooms. Journal of Quality Technology. 2009; 41: 95-109.

[10] Zafar S, Fauzia K, Khan M. Standardization of Anaesthesia Ready Time and reasons of delay in induction of anaesthesia. J Pak Med Assoc. 2006; 56: 112-115.

[11] Mohinder K, Malhotra S. Reasons for delay in turnover time in OR- an observational study. Bangladesh Journal of Medical Science. 2017; 16: 245-251.

[12] Malhotra S, Mohinder K, Bhatia K. Study of Induction Times of Various Anaesthetic Techniques. Sch. J. App. Med. 2016; 4: 1756-1763.

[13] Babita G, Agrawal P, D'souza N, Soni K. Start time delays in OR: Different perspectives. Saudi J Anaesth. 2011; 5: 286-288.

[14] Meyers N. Patient-Specific Factors Associated With Surgical Delay In A Large Academic Hospital. Dissertations. University of San Diego. 2019.

[15] Wong J, Khu KJ, Kaderali Z, Bernstein M. Delays in the operating room: signs of an imperfect system. Can J Surg. 2010; 53:189-195.

[16] Jafari M. Reducing Turnover Time to Improve Efficiency in the Operating Room .Master's Projects and Capstones. University of San Francisco. 2017. 